\title{
Does non-invasive brain stimulation applied over the dorsolateral prefrontal cortex non-specifically influence mood and emotional processing in healthy individuals?
}

\author{
Marine Mondino, François Thiffault and Shirley Fecteau * \\ Centre Interdisciplinaire de Recherche en Réadaptation et en Intégration Sociale, Centre de Recherche de I'Institut \\ Universitaire en Santé Mentale de Québec, Faculté de Médecine, Université Laval, Québec City, QC, Canada
}

OPEN ACCESS

Edited by:

Michael A. Nitsche,

Georg-August-University, Germany

Reviewed by:

Vincent Clark

University of New Mexico, USA

Christian Plewnia,

University of Tübingen, Germany

${ }^{*}$ Correspondence:

Shirley Fecteau,

Université Laval, 2325 rue de

I'Université, Quebec City, QC G1V

OA6, Canada

shirley.fecteau@fmed.ulaval.ca

Received: 21 July 2015 Accepted: 22 September 2015 Published: 14 October 2015

Citation:

Mondino M, Thiffault $F$ and Fecteau S (2015) Does non-invasive brain

stimulation applied over the dorsolateral prefrontal cortex

non-specifically influence mood and emotional processing in healthy

individuals?

Front. Cell. Neurosci. 9:399. doi: 10.3389/fncel.2015.00399
The dorsolateral prefrontal cortex (DLPFC) is often targeted with non-invasive brain stimulation (NIBS) to modulate in vivo human behaviors. This brain region plays a key role in mood, emotional processing, and attentional processing of emotional information. In this article, we ask the question: when we target the DLPFC with NIBS, do we modulate these processes altogether, non-specifically, or can we modulate them selectively? We thus review articles investigating the effects of NIBS applied over the DLPFC on mood, emotional processing, and attentional processing of emotional stimuli in healthy subjects. We discuss that NIBS over the DLPFC can modulate emotional processing and attentional processing of emotional stimuli, without specifically influencing mood. Indeed, there seems to be a lack of evidence that NIBS over the DLPFC influences mood in healthy individuals. Finally, there appears to be a hemispheric lateralization: when applied over the left DLPFC, NIBS improved processing of positive stimuli and reduced selective attention for stimuli expressing anger, whereas when applied over the right DLPFC, it increased selective attention for stimuli expressing anger.

Keywords: repetitive transcranial magnetic stimulation, rTMS, transcranial direct current stimulation, tDCS, mood, emotion, attentional processing, dorsolateral prefrontal cortex

\section{Introduction}

Non-invasive brain stimulation techniques (NIBS) such as repetitive transcranial magnetic stimulation (rTMS) and transcranial direct current stimulation (tDCS) can modulate human brain activity and connectivity (Shafi et al., 2012) and selectively improve or disrupt behaviors (Bikson and Rahman, 2013). They are increasingly used and one region that is often targeted with NIBS to modulate human behaviors in vivo is the dorsolateral prefrontal cortex (DLPFC).

Neuroimaging literature reports that the DLPFC plays a key role in mood (Davidson and Irwin, 1999), emotional processing (Herrington et al., 2005) and attentional processing of emotional information (Jacob et al., 2014). A meta-analysis however indicated that mood predominantly elicits activity in the medial inferior PFC, whereas attentional processing of emotional information mainly evokes activity in the DLPFC (Steele and Lawrie, 2004). Hemispherical specialization of emotional processing has also been proposed: activation in the left DLPFC has been associated with positive mood and processing positive stimuli, whereas activation in the right DLPFC has 
been linked to negative mood and processing negative stimuli (Canli et al., 1998).

Importantly, behavioral studies showed that mood, emotional processing, and attention can influence one another in healthy individuals. Experimentally induced depressed mood impaired identification of facial expressions and retrieving negative stimuli (Chepenik et al., 2007) and influenced rating of facial expressions as more negative (Bouhuys et al., 1995). Elevating mood improved implicit processing of happy faces (Quarto et al., 2014). Also, inducing negative attentional bias increased sadness (MacLeod et al., 2002).

Considering the importance of the DLPFC in mood, emotional processing, and attention and the interplay between these processes, when modulating its activity and connectivity with NIBS, one may wonder whether we are influencing these processes altogether non-specifically or whether we can modify them selectively depending on the targeted hemisphere and/or stimulation parameters. Here we aim at reviewing studies applying NIBS over the DLPFC of healthy subjects to modulate (1) mood, (2) emotional processing, and (3) attentional processing of emotional stimuli to decipher these in the interplay between these processes. Here, mood is defined as the emotional state. Emotional processing refers to processing the emotional content of stimuli (e.g., identification of facial expressions, perception of valence, and retrieval of emotional information). Attentional processing of emotional information refers to the attentional processes selecting and prioritizing relevant emotional stimuli.

\section{Can NIBS Applied Over the DLPFC Modulate Mood in Healthy Individuals?}

Studies investigated whether NIBS applied over the DLPFC can modulate mood in healthy individuals (Table 1A). Pascual-Leone et al. (1996) reported that subjects rated higher anxiety and sadness levels and lower happiness level, as assessed with a 5 -item visual analog scale (VAS), after receiving $10 \mathrm{~Hz}$ rTMS over the left DLPFC. Similarly, George et al. (1996) found that subjects rated lower happiness level after $5 \mathrm{~Hz}$ rTMS over the left DLPFC and lower sadness level after rTMS over the right DLPFC. Mood was assessed with a modified version of the NIMH mood scale. Schaller et al. (2011) found elevated mood after delivering nine sessions of $25 \mathrm{~Hz}$ rTMS over the left DLPFC when measured with the Beck Depression Inventory (BDI), but not with a 6-item VAS. Contrarily to these studies, others reported no significant mood change with high-frequency rTMS over the DLPFC. Mood assessed with 5-item VAS was not modulated with $20 \mathrm{~Hz}$ over the left DLPFC (Mosimann et al., 2000) or $10 \mathrm{~Hz}$ rTMS over the left or right DLPFC (De Raedt et al., 2010). Similarly, mood was not changed with $10 \mathrm{~Hz}$ over the left DLPFC as assessed with VAS and the Profile of Mood Scale (POMS) (Baeken et al., 2006) or $10 \mathrm{~Hz}$ over the right DLPFC as tested with the POMS (Baeken et al., 2010; Vanderhasselt et al., 2011), VAS or the Positive and Negative Affect Schedule (PANAS) (Baeken et al., 2008). Padberg et al. (2001) reported that $10 \mathrm{~Hz}$ rTMS over the left or right DLPFC had no impact on mood assessed by an 8-item VAS, however when targeting the left DLPFC subjects displayed increased frequency and shorter reaction times of laughing reactions when presented with funny stimuli. Hoy et al. (2010) combined $5 \mathrm{~Hz}$ rTMS over the left DLPFC with exposition of positive stimuli to modulate mood. This combination or either method alone (rTMS or exposition) had no impact on mood assessed by a 5-item VAS and an affective go-no-go task. Some studies used low-frequency rTMS to investigate mood. Mood was not influenced with $1 \mathrm{~Hz}$ over the left or right DLPFC as assessed with a 4-item VAS (Grisaru et al., 2001) or $0.6 \mathrm{~Hz}$ rTMS over the left or right DLPFC as tested with the POMS (d'Alfonso et al., 2000). Anxiety measured by the State-Trait Anxiety Inventory was reduced after subjects received $1 \mathrm{~Hz}$ rTMS over the right DLPFC (Schutter et al., 2001).

In regards to tDCS, anodal tDCS over the left dlPFC and cathodal over the right deltoid muscle suppressed upset induced by the Paced Auditory Serial Addition Task (Plewnia et al., 2015). There seems to be no other studies reporting significant changes in mood when targeting the DLPFC of healthy subjects. This has been tested with anodal over the left and cathodal over the right DLPFC or the reverse electrode montage (Plazier et al., 2012; Morgan et al., 2014), anodal over the left DLPFC and cathodal over the right supraorbital region or the reverse montage (Nitsche et al., 2012), anodal over the left DLPFC and cathodal over the primary motor cortex (M1) or the reverse electrode montage (Peña-Gómez et al., 2011), and anodal over the left DLPFC and cathodal over the right supraorbital region delivering four sessions (Motohashi et al., 2013).

In sum, most studies reported that NIBS does not significantly influence mood in healthy subjects. Those reporting positive findings indicated a hemispheric lateralization: targeting the left DLPFC induced both negative and positive mood, whereas targeting the right DLPFC elevated mood.

\section{Can NIBS Applied Over the DLPFC Modulate Emotional Processing in Healthy Individuals?}

Studies applied NIBS over the DLPFC of healthy individuals to investigate emotional processing, especially perception of valence, identification of facial expressions, and retrieval of emotional information (Table 1B). In regards to perception of valence, Peña-Gómez et al. (2011) found that negative stimuli were perceived as less negative after anodal tDCS over the left DLPFC and cathodal over the right M1. Furthermore, this effect was stronger in subjects with higher subclinical scores of introversion.

For identification of facial expressions, Nitsche et al. (2012) reported that subjects were faster at identifying faces expressing positive and negative emotions during anodal or cathodal tDCS over the left DLPFC, with greater effect during anodal tDCS and positive stimuli. Conson et al. (2015) found that healthy men, but not women, were faster at recognizing fearful faces after receiving anodal and cathodal tDCS over the right and left DLPFC, respectively. 
TABLE 1 | Summaries of the studies investigating the effects of non-invasive brain stimulation applied over the dorsolateral prefrontal cortex on mood, emotional processing, and attentional processing of emotional information in healthy individuals.

\begin{tabular}{|c|c|c|c|c|c|c|c|}
\hline References & Study design & N (Males) & $\begin{array}{l}\text { Mean age } \\
\text { in years }\end{array}$ & $\begin{array}{l}\text { Site of } \\
\text { stimulation* }\end{array}$ & NIBS parameters ${ }^{\star \star}$ & $\begin{array}{l}\text { Experimental outcomes } \\
\text { (Time of assessment) }\end{array}$ & Main results \\
\hline \multicolumn{8}{|c|}{ (A) EFFECTS OF NIBS ON MOOD } \\
\hline \multicolumn{8}{|l|}{ rTMS studies } \\
\hline $\begin{array}{l}\text { Schaller et al., } \\
2011\end{array}$ & $\begin{array}{l}\text { Parallel } \\
\text { Sham controlled } \\
9 \text { rTMS sessions }\end{array}$ & $44(44)$ & $\begin{array}{l}\text { Range: } \\
\text { 19-33 }\end{array}$ & $\begin{array}{l}\text { L DLPFC } \\
\text { (5 cm anterior } \\
\text { to } \mathrm{M} 1)\end{array}$ & $\begin{array}{l}25 \mathrm{~Hz} \\
15 \text { trains of } 2 \mathrm{~s} \\
8 \mathrm{~s} \mathrm{ITI} \\
750 \text { pulses } \\
\text { Increasing MT across } \\
\text { sessions (from } 100 \text { to } \\
136.9 \% \text { ) }\end{array}$ & $\begin{array}{l}\text { 1. BDI } \\
\text { 2. } 6 \text {-item mood VAS: } \\
\text { happy/unhappy, cheerful/sad, } \\
\text { energetic/lack of energy, } \\
\text { lively/gloomy, } \\
\text { even-tempered/restless, } \\
\text { serious/smiling } \\
\text { (day } 0 \text {, day } 5 \text {, day 9) }\end{array}$ & $\begin{array}{l}\text { Active vs. sham rTMS: } \\
\text { 1. BDI: Reduced sum scores } \\
\text { and scores on "libido", } \\
\text { "fatigability" and "weight } \\
\text { loss" at day } 5 \text { and } 9 \\
\text { 2. Mood VAS: No effect }\end{array}$ \\
\hline \multirow[t]{2}{*}{$\begin{array}{l}\text { Baeken et al., } \\
2010\end{array}$} & $\begin{array}{l}\text { Parallel } \\
\text { No sham }\end{array}$ & $10(0)$ & N/A & $\begin{array}{l}\text { L DLPFC } \\
(\mathrm{MRI} B N)\end{array}$ & $\begin{array}{l}10 \mathrm{~Hz} \\
40 \text { trains of } 3.9 \mathrm{~s} \\
26.1 \mathrm{~s} \mathrm{ITI} \\
1560 \text { pulses } \\
100 \% \mathrm{MT}\end{array}$ & 1. POMS-32 (t0, t1) & $\begin{array}{l}\text { Before vs. after active L } \\
\text { DLPFC rTMS: } \\
\text { 1. POMS-32: No effect } \\
\text { Before vs. after active } R \\
\text { DLPFC rTMS: } \\
\text { 1. POMS-32: No effect }\end{array}$ \\
\hline & & $10(0)$ & $\mathrm{N} / \mathrm{A}$ & $\begin{array}{l}\text { R DLPFC } \\
(\mathrm{MRI} B N)\end{array}$ & & & \\
\hline $\begin{array}{l}\text { Hoy et al., } \\
2010\end{array}$ & $\begin{array}{l}\text { Crossover } \\
\text { Sham controlled } \\
\text { rTMS combined } \\
\text { with exposure to } \\
\text { positive or } \\
\text { neutral pictures }\end{array}$ & $10(4)$ & 31.2 & $\begin{array}{l}\text { L DLPFC } \\
\text { (10/20 EEG) }\end{array}$ & $\begin{array}{l}5 \mathrm{~Hz} \\
30 \text { trains of } 10 \mathrm{~s} \\
20 \mathrm{~s} \mathrm{ITI} \\
1500 \text { pulses } \\
120 \% \mathrm{MT}\end{array}$ & $\begin{array}{l}\text { 1. AGN task with happy and } \\
\text { sad words } \\
\text { 2. } 5 \text {-item mood VAS: } \\
\text { sadness, happiness, } \\
\text { tiredness, anxiety, } \\
\text { pain-discomfort } \\
\text { 3. Valence and arousal ratings } \\
\text { on IAPS pictures (t0, } \mathrm{t} 1)\end{array}$ & $\begin{array}{l}\text { Active vs. sham rTMS: } \\
\text { 1. AGN task: No effect } \\
\text { 2. Mood VAS: No effect } \\
\text { 3. Valence and arousal } \\
\text { ratings: No effect }\end{array}$ \\
\hline \multirow[t]{2}{*}{$\begin{array}{l}\text { Baeken et al., } \\
2008\end{array}$} & $\begin{array}{l}\text { Crossover } \\
\text { Sham controlled }\end{array}$ & $27(0)$ & 25.2 & $\begin{array}{l}\text { R DLPFC } \\
(\mathrm{MRI} B N)\end{array}$ & $\begin{array}{l}10 \mathrm{~Hz} \\
40 \text { trains of } 4.9 \mathrm{~s} \\
26.1 \mathrm{~s} \mathrm{ITI} \\
1560 \text { pulses } \\
110 \% \mathrm{MT}\end{array}$ & $\begin{array}{l}\text { 1. 5-item mood VAS: } \\
\text { sadness, tension, vigor, anger, } \\
\text { tiredness } \\
\text { 2. POMS-32 (to, t1, t30) }\end{array}$ & $\begin{array}{l}\text { Active R DLPFC vs. sham } \\
\text { rTMS: } \\
\text { 1. Mood VAS: No effect } \\
\text { 2. POMS-32: No effect }\end{array}$ \\
\hline & $\begin{array}{l}\text { Crossover } \\
\text { Sham controlled }\end{array}$ & $20(0)$ & 25.6 & $\begin{array}{l}\text { L DLPFC } \\
(\mathrm{MRI} B N)\end{array}$ & $\begin{array}{l}10 \mathrm{~Hz} \\
40 \text { trains of } 4.9 \mathrm{~s} \\
26.1 \mathrm{~s} \mathrm{ITI} \\
1560 \text { pulses } \\
110 \% \mathrm{MT}\end{array}$ & $\begin{array}{l}\text { 1. 5-item mood VAS: } \\
\text { sadness, tension, vigor, anger, } \\
\text { tiredness } \\
\text { 2. POMS-32 (to, } \mathrm{t} 1, \mathrm{t} 30)\end{array}$ & $\begin{array}{l}\text { Active L DLPFC vs. sham } \\
\text { rTMS: } \\
\text { 1. Mood VAS: No effect } \\
\text { 2. POMS-32: No effect }\end{array}$ \\
\hline $\begin{array}{l}\text { Baeken et al., } \\
2006\end{array}$ & $\begin{array}{l}\text { Crossover } \\
\text { Sham controlled }\end{array}$ & $28(0)$ & 28.7 & $\begin{array}{l}\text { L DLPFC } \\
(\mathrm{MRI} B N)\end{array}$ & $\begin{array}{l}10 \mathrm{~Hz} \\
40 \text { trains of } 3.9 \mathrm{~s} \\
26.1 \mathrm{~s} \mathrm{ITI} \\
1560 \text { pulses } \\
110 \% \mathrm{MT}\end{array}$ & $\begin{array}{l}\text { 1. 5-item mood VAS: } \\
\text { sadness, tension, vigor, anger, } \\
\text { tiredness } \\
\text { 2. POMS-32 } \\
\text { (t0, t1, t30) }\end{array}$ & $\begin{array}{l}\text { Active vs. sham rTMS: } \\
\text { 1. Mood VAS: No effect } \\
\text { 2. POMS-32: No effect }\end{array}$ \\
\hline $\begin{array}{l}\text { Grisaru et al., } \\
2001\end{array}$ & $\begin{array}{l}\text { Crossover } \\
\text { Sham controlled }\end{array}$ & $18(7)$ & 40.5 & $\begin{array}{l}\text { L DLPFC } \\
\text { R DLPFC } \\
\text { ( } 5 \mathrm{~cm} \text { anterior } \\
\text { to } \mathrm{M} 1 \text { or } \mathrm{M} 2)\end{array}$ & $\begin{array}{l}1 \mathrm{~Hz} \\
1 \text { single train } \\
500 \text { pulses } \\
110 \% \mathrm{MT}\end{array}$ & $\begin{array}{l}\text { 1. 4-item mood VAS: } \\
\text { irritability, anxiety, depression, } \\
\text { happiness } \\
\text { (to, t5, t10, t30, t240) }\end{array}$ & $\begin{array}{l}\text { Active (either L or R DLPFC) } \\
\text { vs. sham rTMS: } \\
\text { 1. Mood VAS: No effect }\end{array}$ \\
\hline $\begin{array}{l}\text { Padberg } \\
\text { et al., } 2001\end{array}$ & $\begin{array}{l}\text { Crossover } \\
\text { No sham }\end{array}$ & $9(5)$ & 29.8 & $\begin{array}{l}\text { L DLPFC } \\
\text { R DLPFC } \\
\text { ( } 5 \mathrm{~cm} \text { anterior } \\
\text { to } \mathrm{M} 1 \text { or } \mathrm{M} 2)\end{array}$ & $\begin{array}{l}10 \mathrm{~Hz} \\
10 \text { trains of } 5 \mathrm{~s} \\
30 \mathrm{~s} \mathrm{ITI} \\
500 \text { pulses } \\
110 \% \mathrm{MT}\end{array}$ & $\begin{array}{l}\text { 1. 8-item mood VAS: mood, } \\
\text { emotion, general state, } \\
\text { anxiety, activity, physical } \\
\text { condition, self-perception (t0, } \\
\text { t1, t15) } \\
\text { 2. Facial expressions } \\
\text { recording with ultrasonic } \\
\text { signal emitted by mouth and } \\
\text { eyes muscles during a funny } \\
\text { movie (t0, t1) }\end{array}$ & $\begin{array}{l}\text { Active rTMS, L vs. R DLPFC: } \\
\text { 1. Mood VAS: No effect } \\
\text { 2. Facial expressions: } \\
\text { Increased frequencies of } \\
\text { laughing and shorter RT of } \\
\text { laughing movements }\end{array}$ \\
\hline
\end{tabular}


TABLE 1 | Continued

\begin{tabular}{|c|c|c|c|c|c|c|c|}
\hline References & Study design & N (Males) & $\begin{array}{l}\text { Mean age } \\
\text { in years }\end{array}$ & $\begin{array}{l}\text { Site of } \\
\text { stimulation* }\end{array}$ & NIBS parameters ${ }^{\star *}$ & $\begin{array}{l}\text { Experimental outcomes } \\
\text { (Time of assessment) }\end{array}$ & Main results \\
\hline $\begin{array}{l}\text { Schutter } \\
\text { et al., } 2001\end{array}$ & $\begin{array}{l}\text { Crossover } \\
\text { Sham controlled }\end{array}$ & $12(8)$ & 28.4 & $\begin{array}{l}\text { R DLPFC } \\
(10 / 20 \text { EEG) }\end{array}$ & $\begin{array}{l}1 \mathrm{~Hz} \\
1 \text { single train } \\
1200 \text { pulses } \\
130 \% \text { MT }\end{array}$ & $\begin{array}{l}\text { 1. STAI } \\
\text { 2. STAS } \\
\text { (to, } \mathrm{t} 1, \mathrm{t} 35, \mathrm{t} 65)\end{array}$ & $\begin{array}{l}\text { Active vs. sham rTMS: } \\
\text { 1. STAl: Reduced anxiety } \\
\text { 2. STAS: No effect }\end{array}$ \\
\hline $\begin{array}{l}\text { Mosimann } \\
\text { et al., } 2000\end{array}$ & $\begin{array}{l}\text { Crossover } \\
\text { Sham controlled }\end{array}$ & $25(25)$ & 22.4 & $\begin{array}{l}\text { LPFC } \\
(5 \mathrm{~cm} \text { anterior, } \\
2 \mathrm{~cm} \text { lateral to } \\
\text { M1) }\end{array}$ & $\begin{array}{l}20 \mathrm{~Hz} \\
40 \text { trains of } 2 \mathrm{~s} \\
30 \mathrm{~s} \mathrm{ITI} \\
1600 \text { pulses } \\
100 \% \mathrm{MT}\end{array}$ & $\begin{array}{l}\text { 1. 5-item mood VAS: } \\
\text { tiredness, happiness, } \\
\text { sadness, pain, anxiety (to, t20) }\end{array}$ & $\begin{array}{l}\text { Active vs. sham rTMS: } \\
\text { 1. Mood VAS: No effect }\end{array}$ \\
\hline $\begin{array}{l}\text { George et al., } \\
1996\end{array}$ & $\begin{array}{l}\text { Crossover } \\
\text { No sham }\end{array}$ & $10(6)$ & 35 & $\begin{array}{l}\text { L DLPFC } \\
\text { R DLPFC } \\
\text { (5 cm anterior } \\
\text { to } \mathrm{M} 1 \text { or } \mathrm{M} 2 \text { ) }\end{array}$ & $\begin{array}{l}5 \mathrm{~Hz} \\
10 \text { trains of } 10 \mathrm{~s} \\
1 \mathrm{~s} \mathrm{ITI} \\
500 \text { pulses } \\
120 \% \mathrm{MT}\end{array}$ & $\begin{array}{l}\text { 1. NIMH mood scale } \\
\text { 2. Forced-choice mood VAS } \\
\text { 3. PANAS } \\
\text { (to, t30, t60, t90, t180, t480, } \\
\mathrm{t} 1440)\end{array}$ & $\begin{array}{l}\text { Active rTMS, L vs. R DLPFC: } \\
\text { 1. NIMH mood scale: } \\
\text { Reduced happiness and } \\
\text { increased sadness } \\
\text { 2. Forced-choice } \\
\text { mood-VAS: No effect } \\
\text { 3. PANAS: No effect } \\
\text { Active rTMS, R vs. L DLPFC: } \\
\text { 1. NIMH mood scale: } \\
\text { Reduced sadness and } \\
\text { increased happiness } \\
\text { 2. Forced-choice } \\
\text { mood-VAS: No effect } \\
\text { 3. PANAS: No effect }\end{array}$ \\
\hline $\begin{array}{l}\text { Pascual- } \\
\text { Leone et al., } \\
1996\end{array}$ & $\begin{array}{l}\text { Crossover } \\
\text { No sham }\end{array}$ & $10(4)$ & $\begin{array}{l}\text { Range: } \\
22-27\end{array}$ & $\begin{array}{l}\text { L DLPFC } \\
\text { R DLPFC } \\
\text { ( } 5 \mathrm{~cm} \text { anterior } \\
\text { to M1 or M2) } \\
\text { Mid PFC }\end{array}$ & $\begin{array}{l}10 \mathrm{~Hz} \\
10 \text { trains of } 10 \mathrm{~s} \\
25 \mathrm{~s} \mathrm{ITI} \\
500 \text { pulses } \\
110 \% \mathrm{MT}\end{array}$ & $\begin{array}{l}\text { 1. } 5 \text {-item mood VAS: pain } \\
\text { discomfort, sadness, } \\
\text { happiness, anxiety, tiredness } \\
\text { (to, t1) }\end{array}$ & $\begin{array}{l}\text { Active rTMS, L vs. R DLPFC: } \\
\text { 1. Mood VAS: Decreased } \\
\text { happiness and increased } \\
\text { sadness } \\
\text { Active rTMS, L vs. Mid PFC: } \\
\text { 1. Mood VAS: Increased } \\
\text { pain/discomfort, anxiety and } \\
\text { sadness } \\
\text { Active rTMS, R vs. L DLPFC: } \\
\text { 1. Mood VAS: Increased } \\
\text { happiness }\end{array}$ \\
\hline
\end{tabular}

\section{tDCS studies}

\begin{tabular}{|c|c|c|c|c|c|}
\hline $\begin{array}{l}\text { Plewnia et al., } \\
2015\end{array}$ & $\begin{array}{l}\text { Parallel } \\
\text { Sham controlled }\end{array}$ & $28(28)$ & 27.9 & $\begin{array}{l}\text { L DLPFC/R } \\
\text { deltoid } \\
\text { (10/20 EEG) }\end{array}$ & $\begin{array}{l}1 \mathrm{~mA} \\
20 \mathrm{~min} \\
35 \mathrm{~cm}^{2}\end{array}$ \\
\hline
\end{tabular}

\begin{tabular}{|c|c|c|c|c|c|c|c|}
\hline & & & & & & & $\begin{array}{l}\text { active tDCS } \\
\text { 2. PASAT: Shorter } \\
\text { inter-stimulus interval after } \\
\text { anodal vs. sham tDCS } \\
\text { Slower inter-stimulus interval } \\
\text { were correlated to increased } \\
\text { upset }\end{array}$ \\
\hline $\begin{array}{l}\text { Morgan et al., } \\
2014\end{array}$ & $\begin{array}{l}\text { Crossover } \\
\text { No sham }\end{array}$ & $18(9)$ & 23.2 & $\begin{array}{l}\text { L DLPFC/R } \\
\text { DLPFC } \\
\text { R DLPFC/L } \\
\text { DLPFC } \\
(10 / 20 \text { EEG) }\end{array}$ & $\begin{array}{l}1 \mathrm{~mA} \\
12 \mathrm{~min} \\
9 \mathrm{~cm}^{2}\end{array}$ & $\begin{array}{l}\text { 1. PANAS } \\
\text { 2. Motivational state } \\
\text { questionnaire } \\
\text { 3. Memory task with IAPS } \\
\text { pictures }(\mathrm{t} 0, \mathrm{t} 1 \text { ) }\end{array}$ & $\begin{array}{l}\text { Active tDCS, L DLPFC/R } \\
\text { DLPFC vs. R DLPFC/L } \\
\text { DLPFC: } \\
\text { 1. PANAS: No effect } \\
\text { 2. Motivational state } \\
\text { questionnaire: No effect } \\
\text { 3. Memory task: No effect }\end{array}$ \\
\hline $\begin{array}{l}\text { Motohashi } \\
\text { tt al., } 2013\end{array}$ & $\begin{array}{l}\text { Crossover } \\
\text { Sham controlled } \\
4 \text { tDCS sessions }\end{array}$ & 12 (12) & 22 & $\begin{array}{l}\text { L DLPFC/ } \\
\text { supraorbital } \\
\text { region } \\
\text { (10/20 EEG) }\end{array}$ & $\begin{array}{l}1 \mathrm{~mA} \\
20 \mathrm{~min} \\
35 \mathrm{~cm}^{2}\end{array}$ & $\begin{array}{l}\text { 1. POMS-30 } \\
\text { (day } 0 \text {, day } 4 \text { ) }\end{array}$ & $\begin{array}{l}\text { Active vs. sham tDCS: } \\
\text { 1. POMS-30: No effect }\end{array}$ \\
\hline
\end{tabular}


TABLE 1 | Continued

\begin{tabular}{|c|c|c|c|c|c|c|c|}
\hline References & Study design & N (Males) & $\begin{array}{l}\text { Mean age } \\
\text { in years }\end{array}$ & $\begin{array}{l}\text { Site of } \\
\text { stimulation* }\end{array}$ & NIBS parameters ${ }^{\star \star}$ & $\begin{array}{l}\text { Experimental outcomes } \\
\text { (Time of assessment) }\end{array}$ & Main results \\
\hline $\begin{array}{l}\text { Plazier et al., } \\
2012\end{array}$ & $\begin{array}{l}\text { Crossover } \\
\text { Sham controlled }\end{array}$ & 17 (17) & 21.5 & $\begin{array}{l}\text { R DLPFC/L } \\
\text { DLPFC } \\
\text { L DLPFC/R } \\
\text { DLPFC } \\
\text { O2/O1 } \\
\text { O1/O2 } \\
\text { (10/20 EEG) }\end{array}$ & $\begin{array}{l}1.5 \mathrm{~mA} \\
20 \mathrm{~min} \\
35 \mathrm{~cm}^{2}\end{array}$ & $\begin{array}{l}\text { 1. SUDS } \\
\text { 2. POMS-32 } \\
\text { 3. PANAS } \\
\text { 4. BISBAS } \\
(\mathrm{t} 0, \mathrm{t} 1)\end{array}$ & $\begin{array}{l}\text { Active (either four conditions) } \\
\text { vs. sham tDCS: } \\
\text { 1. SUDS: No effect } \\
\text { 2. POMS-32: No effect } \\
\text { 3. PANAS: No effect } \\
\text { 4. BISBAS: No effect }\end{array}$ \\
\hline
\end{tabular}

(B) EFFECTS OF NIBS ON EMOTIONAL PROCESSING

rTMS studies

$69(31)$

28.1
Balconi and Crossover

Cobelli, 2015 Sham controlled
L DLPFC
PZ

(10/20 EEG)
$5 \mathrm{~Hz}$

90 trains of $1 \mathrm{~s}$

$5 \mathrm{~s}$ ITI

450 pulses

$100 \%$ MT

\begin{tabular}{|c|c|c|c|c|c|c|}
\hline & & & & 年 & $\begin{array}{l}450 \text { pulses } \\
100 \% \mathrm{MT}\end{array}$ & $\begin{array}{l}\text { arousal (t0.5) } \\
2 . \text { Valence and arousal } \\
\text { questionnaire with words and } \\
\text { pictures (t1) }\end{array}$ \\
\hline $\begin{array}{l}\text { Balconi and } \\
\text { Ferrari, } 2013\end{array}$ & $\begin{array}{l}\text { Crossover } \\
\text { Sham controlled }\end{array}$ & 27 (12) & $\begin{array}{l}\text { Range: } \\
\text { 21-36 }\end{array}$ & $\begin{array}{l}\text { L DLPFC } \\
\text { Cz } \\
\text { (10/20 EEG) }\end{array}$ & $\begin{array}{l}5 \mathrm{~Hz} \\
180 \text { trains of } 1 \mathrm{~s} \\
5 \mathrm{~s} \mathrm{ITI} \\
900 \text { pulses } \\
100 \% \mathrm{MT}\end{array}$ & $\begin{array}{l}\text { 1. Memory task with positive } \\
\text { and negative words among } \\
\text { semantically related or } \\
\text { unrelated distractors (to.5) }\end{array}$ \\
\hline
\end{tabular}

1. Memory task with positive and negative words and pictures with high and low arousal (t0.5)

2. Valence and arousal with words and Memory task with positive unrelated distractors (to.5)
Active rTMS, L DLPFC vs. $\mathrm{Pz}$ and sham:

1. Memory task: Increased accuracy and reduced RT for positive high arousal words and pictures 2. Valence and arousal questionnaire: No effect

Active rTMS, L DLPFC vs. $\mathrm{Cz}$ and sham:

1. Memory task: Reduced RT for positive targets and positive (related and unrelated) distractors in subjects with high and low anxiety level

\begin{tabular}{|c|c|c|c|c|c|}
\hline $\begin{array}{l}\text { Balconi and } \\
\text { Ferrari, 2012b }\end{array}$ & $\begin{array}{l}\text { Crossover } \\
\text { Sham controlled }\end{array}$ & 30 (13) & $\begin{array}{l}\text { Range: } \\
\text { 21-31 }\end{array}$ & $\begin{array}{l}\text { L DLPFC } \\
\text { Cz } \\
\text { (10/20 EEG) }\end{array}$ & $\begin{array}{l}5 \mathrm{~Hz} \\
90 \text { trains of } 1 \mathrm{~s} \\
5 \mathrm{~s} \mathrm{ITI} \\
450 \text { pulses } \\
100 \% \mathrm{MT}\end{array}$ \\
\hline
\end{tabular}

1. Memory task with positive and negative words (t0.5)

Active rTMS, L DLPFC vs. $\mathrm{Cz}$ and sham:

1. Memory task: increased accuracy for positive vs. negative words in subjects with high and low anxiety level. Reduced RT for positive vs. negative words in subjects with high anxiety level

\begin{tabular}{|c|c|c|c|c|c|c|c|}
\hline $\begin{array}{l}\text { Balconi and } \\
\text { Ferrari, 2012a }\end{array}$ & $\begin{array}{l}\text { Crossover } \\
\text { Sham controlled }\end{array}$ & 27 & $\begin{array}{l}\text { Range: } \\
\text { 21-37 }\end{array}$ & $\begin{array}{l}\text { L DLPFC } \\
\text { Cz } \\
\text { (10/20 EEG) }\end{array}$ & $\begin{array}{l}5 \mathrm{~Hz} \\
90 \text { trains of } 1 \mathrm{~s} \\
5 \mathrm{~s} \mathrm{ITI} \\
450 \text { pulses } \\
100 \% \mathrm{MT}\end{array}$ & $\begin{array}{l}\text { 1. Memory task with positive } \\
\text { and negative words among } \\
\text { semantically related or } \\
\text { unrelated distractors (to.5) }\end{array}$ & $\begin{array}{l}\text { Active rTMS, L DLPFC vs. } \\
\text { Cz and sham: } \\
\text { 1. Memory task: Reduced } \\
\text { RT for positive vs. negative } \\
\text { words and related vs. } \\
\text { unrelated positive distractors }\end{array}$ \\
\hline
\end{tabular}

\begin{tabular}{|c|c|c|c|c|c|c|c|}
\hline $\begin{array}{l}\text { Conson et al., } \\
2015\end{array}$ & $\begin{array}{l}\text { Crossover } \\
\text { Sham controlled }\end{array}$ & $16(8)$ & $\begin{array}{l}\text { Range: } \\
\text { 22-30 }\end{array}$ & $\begin{array}{l}\text { L DLPFC/R } \\
\text { DLPFC } \\
\text { R DLPFC/L } \\
\text { DLPFC } \\
\text { (10/20 EEG) }\end{array}$ & $\begin{array}{l}1 \mathrm{~mA} \\
15 \mathrm{~min} \\
35 \mathrm{~cm}^{2}\end{array}$ & $\begin{array}{l}\text { 1. Recognition of facial } \\
\text { expressions task }\end{array}$ & $\begin{array}{l}\text { Active tDCS, R DLPFC/L } \\
\text { DLPFC vs. L DLPFC/R } \\
\text { DLPFC and sham: } \\
\text { 1. Recognition of facial } \\
\text { expressions task: Reduced } \\
\text { RT for fearful faces in male } \\
\text { but not female subjects }\end{array}$ \\
\hline $\begin{array}{l}\text { Nitsche et al., } \\
2012\end{array}$ & $\begin{array}{l}\text { Crossover } \\
\text { Sham controlled }\end{array}$ & $14(9)$ & 33.3 & $\begin{array}{l}\text { L DLPFC/ } \\
\text { supraorbital } \\
\text { region } \\
\text { Supraorbital } \\
\text { region/L } \\
\text { DLPFC } \\
\text { (10/20 EEG) }\end{array}$ & $\begin{array}{l}1 \mathrm{~mA} \\
20 \mathrm{~min} \\
35 \mathrm{~cm}^{2}\end{array}$ & $\begin{array}{l}\text { 1. 14-item mood VAS } \\
\text { (t0, t15, t30, t45, t60, t120, } \\
\text { t180, t240, t300, following } \\
\text { morning) }\end{array}$ & $\begin{array}{l}\text { Active tDCS, } L \\
\text { DLPFC/supraorbital region } \\
\text { vs. supraorbital region/L } \\
\text { DLPFC and sham: } \\
\text { 1. Mood VAS: No effect }\end{array}$ \\
\hline
\end{tabular}


TABLE 1 | Continued

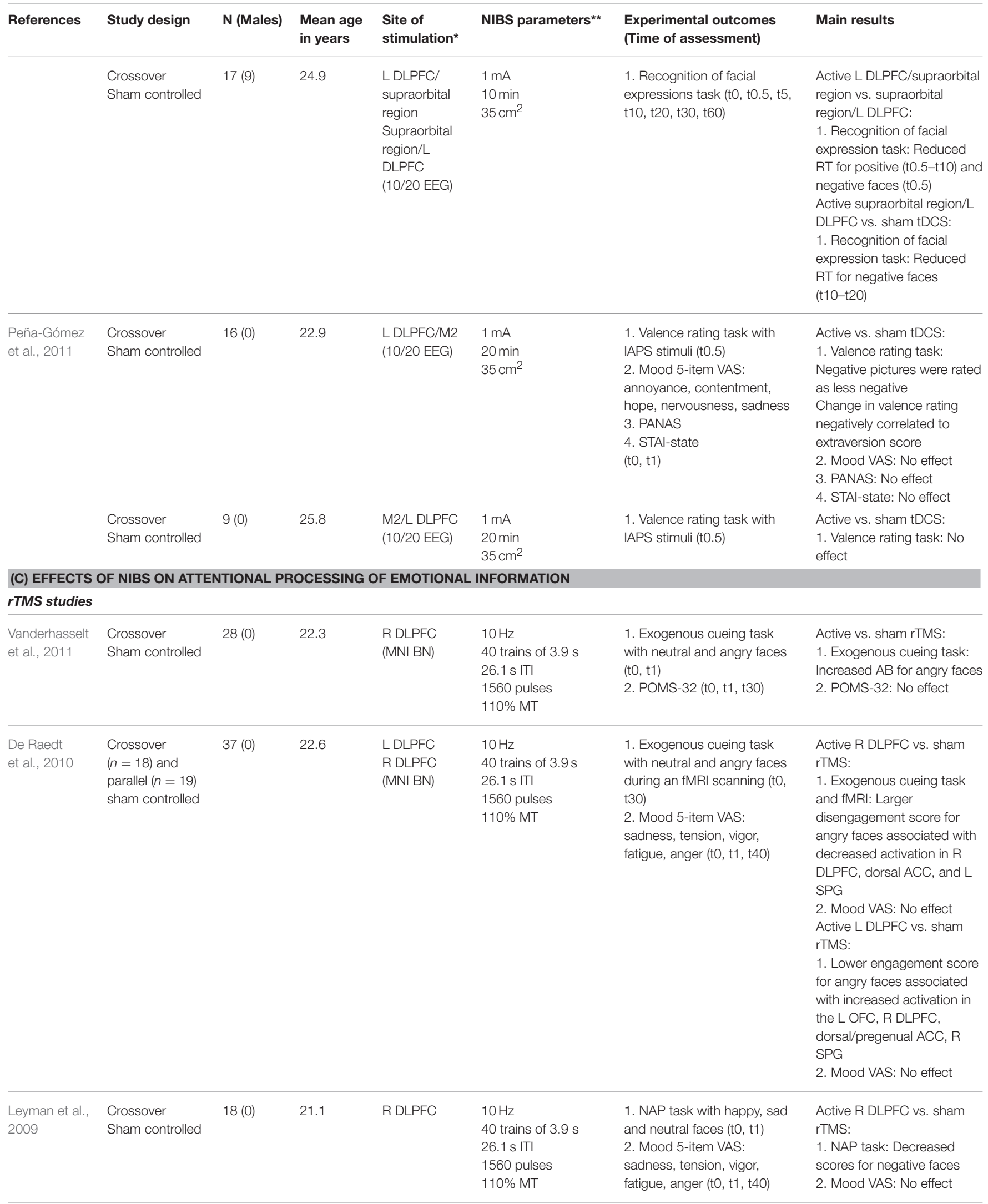


TABLE 1 | Continued

\begin{tabular}{|c|c|c|c|c|c|c|c|}
\hline References & Study design & N (Males) & $\begin{array}{l}\text { Mean age } \\
\text { in years }\end{array}$ & $\begin{array}{l}\text { Site of } \\
\text { stimulation* }\end{array}$ & NIBS parameters ${ }^{\star \star}$ & $\begin{array}{l}\text { Experimental outcomes } \\
\text { (Time of assessment) }\end{array}$ & Main results \\
\hline & $\begin{array}{l}\text { Crossover } \\
\text { Sham controlled }\end{array}$ & $22(0)$ & 24 & $\begin{array}{l}\text { L DLPFC } \\
(\mathrm{MNI} B N)\end{array}$ & $\begin{array}{l}10 \mathrm{~Hz} \\
40 \text { trains of } 3.9 \mathrm{~s} \\
26.1 \mathrm{~s} \mathrm{ITI} \\
1560 \text { pulses } \\
110 \% \mathrm{MT}\end{array}$ & $\begin{array}{l}\text { 1. NAP task with happy, sad } \\
\text { and neutral faces (t0, t1) } \\
\text { 2. Mood 5-item VAS: } \\
\text { sadness, tension, vigor, } \\
\text { fatigue, anger (t0, t1, t40) }\end{array}$ & $\begin{array}{l}\text { Active L DLPFC vs. sham } \\
\text { rTMS: } \\
\text { 1. NAP task: No effect } \\
\text { 2. Mood VAS: No effect }\end{array}$ \\
\hline $\begin{array}{l}\text { Van Honk } \\
\text { et al., 2002b }\end{array}$ & $\begin{array}{l}\text { Crossover } \\
\text { Sham controlled }\end{array}$ & $8(4)$ & $\begin{array}{l}\text { Range: } \\
\text { 20-26 }\end{array}$ & $\begin{array}{l}\text { R DLPFC } \\
(10 / 20 \text { EEG) }\end{array}$ & $\begin{array}{l}1 \mathrm{~Hz} \\
1 \text { single train } \\
1200 \text { pulses } \\
130 \% \mathrm{MT}\end{array}$ & $\begin{array}{l}\text { 1. Emotional Stroop task with } \\
\text { masked and unmasked } \\
\text { neutral and fearful faces (t30) }\end{array}$ & $\begin{array}{l}\text { Active vs. sham rTMS: } \\
\text { 1. Emotional Stroop task: } \\
\text { Decreased attention for } \\
\text { unmasked fearful faces }\end{array}$ \\
\hline $\begin{array}{l}\text { van Honk } \\
\text { et al., } 2002 a\end{array}$ & $\begin{array}{l}\text { Crossover with } \\
\text { no sham }\end{array}$ & $10(0)$ & $\begin{array}{l}\text { Range: } \\
\text { 18-30 }\end{array}$ & $\begin{array}{l}\text { L DLPFC } \\
\text { R DLPFC } \\
\text { ( } 5 \mathrm{~cm} \text { anterior } \\
\text { to } \mathrm{M} 1 \text { or } \mathrm{M} 2)\end{array}$ & $\begin{array}{l}0.6 \mathrm{~Hz} \\
1 \text { single train } \\
540 \text { pulses } \\
130 \% \mathrm{MT}\end{array}$ & $\begin{array}{l}\text { 1. Emotional Stroop task with } \\
\text { neutral and angry faces }(\mathrm{t} 1) \\
\text { 2. PEP (tO, t1) }\end{array}$ & $\begin{array}{l}\text { Active rTMS, R vs. L DLPFC: } \\
\text { 1. Emotional Stroop task: } \\
\text { Increased attention for angry } \\
\text { faces } \\
\text { 2. PEP: Reduced PEP } \\
\text { Correlation between } \\
\text { increased attention and } \\
\text { reduced PEP }\end{array}$ \\
\hline $\begin{array}{l}\text { d'Alfonso } \\
\text { et al., } 2000\end{array}$ & $\begin{array}{l}\text { Crossover } \\
\text { No sham }\end{array}$ & $10(0)$ & $\begin{array}{l}\text { Range: } \\
\text { 18-30 }\end{array}$ & $\begin{array}{l}\text { L DLPFC } \\
\text { R DLPFC } \\
\text { ( } 5 \mathrm{~cm} \text { anterior } \\
\text { to } \mathrm{M} 1 \text { or } \mathrm{M} 2 \text { ) }\end{array}$ & $\begin{array}{l}0.6 \mathrm{~Hz} \\
1 \text { single train } \\
540 \text { pulses } \\
130 \% \mathrm{MT}\end{array}$ & $\begin{array}{l}\text { 1. Emotional Stroop task with } \\
\text { neutral and angry faces (t10) } \\
\text { 2. POMS-32 (t0, t1) }\end{array}$ & $\begin{array}{l}\text { Active rTMS, L vs. R DLPFC: } \\
\text { 1. Emotional Stroop task: } \\
\text { Decreased attention for } \\
\text { angry faces } \\
\text { 2. POMS-32: No effect } \\
\text { Active rTMS, R vs. L DLPFC } \\
\text { 1. Emotional Stroop task: } \\
\text { Increased attention for angry } \\
\text { faces } \\
\text { 2. POMS-32: No effect }\end{array}$ \\
\hline
\end{tabular}

\section{tDCS studies}

\begin{tabular}{|c|c|c|c|c|c|c|c|}
\hline $\begin{array}{l}\text { Wolkenstein } \\
\text { et al., } 2014\end{array}$ & $\begin{array}{l}\text { Crossover } \\
\text { Sham controlled }\end{array}$ & $28(8)$ & 30.9 & $\begin{array}{l}\text { R deltoid/L } \\
\text { DLPFC } \\
\text { (10/20 EEG) }\end{array}$ & $\begin{array}{l}1 \mathrm{~mA} \\
20 \mathrm{~min} \\
35 \mathrm{~cm}^{2}\end{array}$ & $\begin{array}{l}\text { 1. DWM (t0.5) } \\
\text { 2. AIT with positive, neutral } \\
\text { and negative pictures (t1) } \\
\text { 3. PANAS (t0, } t 1)\end{array}$ & $\begin{array}{l}\text { Active vs. sham tDCS: } \\
\text { 1. DWM: Reduced accuracy } \\
\text { for negative vs. neutral and } \\
\text { positive pictures } \\
\text { 2. AIT: Longer RT for } \\
\text { negative vs. neutral and } \\
\text { positive pictures } \\
\text { 3. PANAS: No effect }\end{array}$ \\
\hline $\begin{array}{l}\text { Clarke et al., } \\
2014\end{array}$ & $\begin{array}{l}\text { Parallel } \\
\text { Sham controlled } \\
\text { "Attend threat" } \\
\text { ABM + active } \\
\text { tDCS } \\
\text { "Avoid threat" } \\
\text { ABM + active } \\
\text { tDCS }\end{array}$ & $20(6)$ & 19.6 & $\begin{array}{l}\text { L DLPFC/L } \\
\text { superior } \\
\text { trapezius } \\
\text { (10/20 EEG) }\end{array}$ & $\begin{array}{l}1 \mathrm{~mA} \\
\text { mean } 17 \mathrm{~min} \\
24 \mathrm{~cm}^{2}\end{array}$ & $\begin{array}{l}\text { 1. AB assessment task with } \\
\text { neutral and threatening words } \\
(\mathrm{t} 0, \mathrm{t} 1)\end{array}$ & $\begin{array}{l}\text { "Attend threat" ABM } \\
\text { combined with active tDCS } \\
\text { vs. "attend threat" ABM } \\
\text { combined with sham tDCS: } \\
\text { 1. AB assessment task: } \\
\text { Increased AB to threat } \\
\text { "Avoid threat" ABM } \\
\text { combined with active tDCS } \\
\text { vs. "Avoid threat" combined } \\
\text { with sham tDCS: } \\
\text { 1. AB assessment task: } \\
\text { Decreased AB to threat }\end{array}$ \\
\hline
\end{tabular}


TABLE 1 | Continued

\begin{tabular}{|c|c|c|c|c|c|c|c|}
\hline References & Study design & N (Males) & $\begin{array}{l}\text { Mean age } \\
\text { in years }\end{array}$ & $\begin{array}{l}\text { Site of } \\
\text { stimulation* }^{*}\end{array}$ & NIBS parameters ${ }^{\star \star}$ & $\begin{array}{l}\text { Experimental outcomes } \\
\text { (Time of assessment) }\end{array}$ & Main results \\
\hline & $\begin{array}{l}\text { "Attend threat" } \\
\text { ABM + sham } \\
\text { tDCS }\end{array}$ & $22(7)$ & 20.6 & & & & \\
\hline & $\begin{array}{l}\text { "Avoid threat" } \\
\text { ABM + sham } \\
\text { tDCS }\end{array}$ & $18(8)$ & 19.9 & & & & \\
\hline $\begin{array}{l}\text { Feeser et al., } \\
2014\end{array}$ & $\begin{array}{l}\text { Parallel } \\
\text { sham controlled } \\
\text { tDCS applied } \\
\text { during emotional } \\
\text { regulation (4 } \\
\text { conditions: } \\
\text { maintain neutral } \\
\text { emotions, } \\
\text { downregulate, } \\
\text { upregulate, or } \\
\text { maintain } \\
\text { negative } \\
\text { emotions) }\end{array}$ & $42(20)$ & 28.5 & $\begin{array}{l}\text { R DLFPC/L } \\
\text { supraorbital } \\
\text { region } \\
\text { (10/20 EEG) }\end{array}$ & $\begin{array}{l}1.5 \mathrm{~mA} \\
20 \mathrm{~min} \\
\text { Anodal: } 35 \mathrm{~cm}^{2} \\
\text { Cathodal: } 100 \mathrm{~cm}^{2}\end{array}$ & $\begin{array}{l}\text { 1. Arousal ratings on IAPS } \\
\text { pictures (t0.5) } \\
\text { 2. Skin conductance } \\
\text { response (t0.5) } \\
\text { 3. Gaze fixation (t0.5) } \\
\text { 4. Multidimensional State } \\
\text { Questionnaire }(\mathrm{t} 0, \mathrm{t} 1)\end{array}$ & $\begin{array}{l}\text { Active vs. Sham tDCS: } \\
\text { 1. Arousal ratings: Lower in } \\
\text { the downregulation } \\
\text { conditions. Higher and in the } \\
\text { negative maintain in the } \\
\text { upregulation condition } \\
\text { 2. Skin conductance: Lower } \\
\text { response in the } \\
\text { downregulation condition. } \\
\text { Higher response in the } \\
\text { upregulation condition } \\
\text { 3. Gaze fixation: No effect } \\
\text { 4. Multidimensional State } \\
\text { Questionnaire: No effect }\end{array}$ \\
\hline
\end{tabular}

"Site of stimulation is provided as follows, for rTMS: coil position, for tDCS: anode/cathode position. The method used to define the target is provided as follows, (10/20 EEG), Electrode

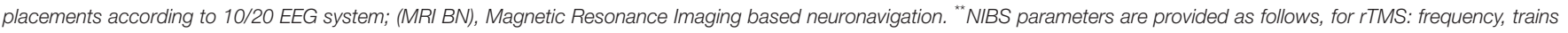

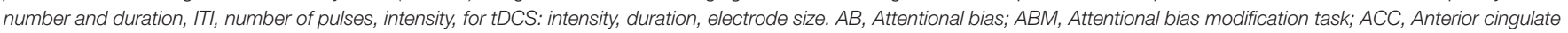

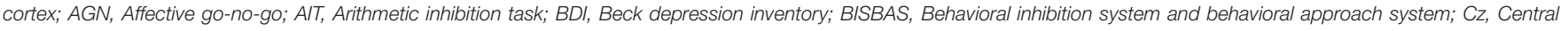

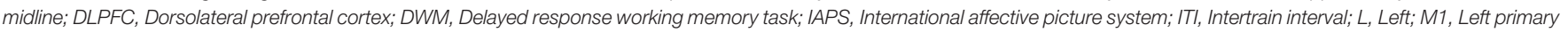

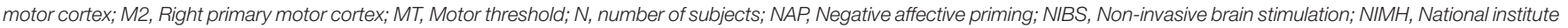

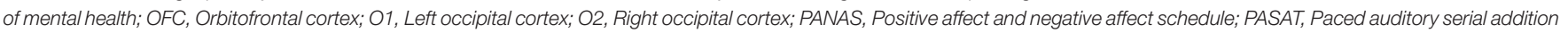

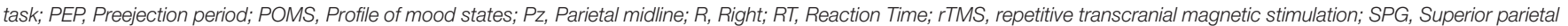

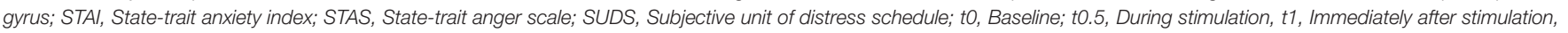
tX, X minutes after stimulation; tDCS, transcranial Direct Current Stimulation; VAS, Visual analog scale.

For retrieval of emotional stimuli, healthy subjects were faster at recognizing positive stimuli (Balconi and Ferrari, 2012a,b, 2013), especially stimuli of high arousal (Balconi and Cobelli, 2015), after receiving $5 \mathrm{~Hz}$ rTMS over the left DLPFC. Morgan et al. (2014) observed no change on retrieval of emotional stimuli delivering anodal and cathodal tDCS over the left and right DLPFC, respectively, or with the reverse montage.

Overall, NIBS targeting the DLPFC, especially the left hemisphere, seems to modulate emotional processing in healthy individuals, such as perceiving negative stimuli as less negative, improving identification of positive stimuli, and enhancing retrieval of positive information.

\section{Can NIBS Applied Over the DLPFC Modulate Attentional Processing of Emotional Information in Healthy Individuals?}

Several studies tested the effects of NIBS over the DLPFC of healthy individuals on attentional processing of emotional information (Table 1C). Selective attention toward emotional information has been tested with high- and low-frequency rTMS over the right and left DLPFC. Attention to angry faces was increased when targeting the right DLPFC with $10 \mathrm{~Hz}$ rTMS (De Raedt et al., 2010; Vanderhasselt et al., 2011) and 0.6 Hz rTMS (d'Alfonso et al., 2000; van Honk et al., 2002a). Interestingly, increased attentional bias toward angry faces was positively correlated with subject's anxiety level (Vanderhasselt et al., 2011) and elevated sympathetic activity (van Honk et al., 2002a). Moreover, targeting the right DLPFC with $1 \mathrm{~Hz}$ rTMS reduced attention to fearful faces (Van Honk et al., 2002b). Attention to angry faces was also reduced with $0.6 \mathrm{~Hz}$ rTMS (d'Alfonso et al., 2000) and $10 \mathrm{~Hz}$ rTMS (De Raedt et al., 2010) when targeting the left DLPFC.

NIBS over the DLPFC has also been used to promote attentional training. Clarke et al. (2014) tested the effects of tDCS during two attention bias modification tasks: one task trains attention to attend threat, whereas the other trains attention to avoid threat. Subjects receiving tDCS with the anode over the left DLPFC and the cathode over the left superior trapezius muscle displayed increased attentional bias to threat when trained to attend threat, but decreased attentional bias to threat when trained to avoid threat.

For inhibitory control of emotional information, it has been shown that targeting the right, but not the left DLPFC with 
$10 \mathrm{~Hz}$ rTMS impaired inhibition of negative stimuli (e.g., reduced negative affective priming for negative stimuli; Leyman et al., 2009). tDCS with the cathode over the left DLPFC and the anode over the right deltoid muscle also impaired inhibitory control for negative but not positive or neutral stimuli (Wolkenstein et al., 2014).

tDCS with the anode over the right DLPFC and the cathode over the left supraorbital region facilitated cognitive reappraisal by increasing emotional responsiveness (arousal rating of negative picture and skin conductance response) during negative emotion upregulation or decreasing emotional responsiveness during negative emotion downregulation (Feeser et al., 2014).

Overall, NIBS seems to modulate attentional processing of emotional stimuli, and these effects seem to depend on the targeted hemisphere. Specifically, selective attention toward anger increased when targeting the right DLPFC, but decreased when targeting the left DLPFC. Also, NIBS over either the left or right DLPFC disrupted inhibitory control when processing negative but not positive or neutral stimuli.

\section{Discussion}

We reviewed here studies investigating the effects of NIBS applied over the DLPFC on mood, emotional processing and attentional processing of emotional information in healthy individuals. Overall, NIBS can selectively modulate processing of emotional information without significantly influencing mood. Specifically, NIBS over the left DLPFC resulted in improving identification of positive facial expressions (Nitsche et al., 2012), rating negative stimuli as less negative (Peña-Gómez et al., 2011), and reducing attention toward anger (d'Alfonso et al., 2000; De Raedt et al., 2010), without influencing mood. NIBS over the right DLPFC increased attention toward anger without influencing mood (d'Alfonso et al., 2000; De Raedt et al., 2010; Vanderhasselt et al., 2011). NIBS applied over either the left or right DLPFC disrupted inhibitory control when processing negative stimuli without interfering with mood (Leyman et al., 2009; Wolkenstein et al., 2014). It remains unclear whether NIBS can significantly influence mood in healthy individuals as several studies reported negative findings (d'Alfonso et al., 2000; Mosimann et al., 2000; Grisaru et al., 2001; Padberg et al., 2001; Baeken et al., 2006, 2008; De Raedt et al., 2010; Hoy et al., 2010; Peña-Gómez et al., 2011; Vanderhasselt et al., 2011; Nitsche et al., 2012; Plazier et al., 2012; Motohashi et al., 2013; Morgan et al., 2014).

We illustrate main findings in Figure 1. We propose that mood, emotional processing, attentional processing of emotional information are closely intertwined like wheels in a gear but that modulation of emotional processing or attention, as it has been induced with NIBS so far, may be insufficient to influence mood in healthy individuals. This is consistent with studies reporting that rTMS over the right DLPFC influenced processing of neutral stimuli such as intentional set switching (Vanderhasselt et al., 2006) and attention (Vanderhasselt et al., 2007) without impacting mood in healthy subjects. Similarly, one session of NIBS over the DLPFC reduced attentional bias for negative stimuli (Brunoni et al., 2014) and inhibitory control impairments independently from mood changes (Vanderhasselt et al., 2009a,b; Wolkenstein and Plewnia, 2013) in individuals with major depressive disorder (MDD). This is also in line with pharmacological work: administering a single dose of antidepressant medication to healthy subjects increased attention to positive words without changes in mood (Browning et al., 2007). Administering 7 days of antidepressant medication to healthy subjects reduced attention to fearful faces (Murphy et al., 2009), impaired identification of negative facial expressions and improved retrieval of positive stimuli (Harmer et al., 2004) without changes in mood.

Findings reviewed here support the hypothesis of hemispheric lateralization in processing emotional information. Neuroimaging studies showed that the left and right DLPFC are specialized in processing positive and negative emotions, respectively (Canli et al., 1998). As schematized in Figure 1, NIBS over the left DLPFC improved processing of positive stimuli and reduced attentional bias for negative stimuli, whereas NIBS over the right DLPFC improved identification of negative stimuli and increased attentional bias for negative stimuli.

In regards to stimulation parameters, it is not clear whether some are more effective than others to modulate mood, emotional processing or attentional processing of emotional stimuli in terms of rTMS frequencies (ranging from 0.6 to $25 \mathrm{~Hz}$ ) or number of pulses (ranging from 450 to 1800 pulses). Higher intensity may induce greater effects: among the six studies using $100 \%$ of motor threshold (MT), four had positive results (all improved retrieval of emotional stimuli) and two had negative findings (no mood change), whereas the five studies using 130\% of MT reported changes in mood and attention. Of note, Schaller et al. (2011) increased intensity from 100 to $130 \%$ of MT, along with the number of sessions, and reported no correlation between intensity and mood changes. For tDCS, anodal may induce greater effects than cathodal on emotional processing (Peña-Gómez et al., 2011; Nitsche et al., 2012), whereas it is not clear whether amplitude (ranging from 1 to $1.5 \mathrm{~mA}$ ) or duration (ranging from 10 to $20 \mathrm{~min}$ ) play an important role on these processes.

Some methodological considerations should be noted. First, mood, emotional processing, and attention to emotional information have been tested with various approaches and outcomes. For instance, mood has been assessed with self-rated homemade VAS on limited number of items (ranging from 4 to 14) to standardized questionnaires (POMS, PANAS), including clinical tools (BDI), whereas emotional processing and attention have been mainly measured in terms of accuracy (percent of correct answers) and response time (changes in milliseconds). These assessments and outcomes may not have the same sensitivity to capture NIBS-induced changes. As an example, Schaller et al. (2011) showed an effect on mood when assessed by the BDI but not by the 6-item VAS. The VAS (as well as the POMS and PANAS) require to rate mood on adjectives (e.g., delighted, timid) with no specific context, whereas the BDI consists of specific questions using contexts to assess mood. Second, NIBSinduced changes have been measured by comparing various NIBS conditions. Some found changes by comparing two active NIBS conditions (e.g., targeting the right vs. left DLPFC) and others used sham conditions that are considered as partially active (e.g., 


\section{NIBS applied over the left DLPFC}

\section{NIBS applied over the}

right DLPFC

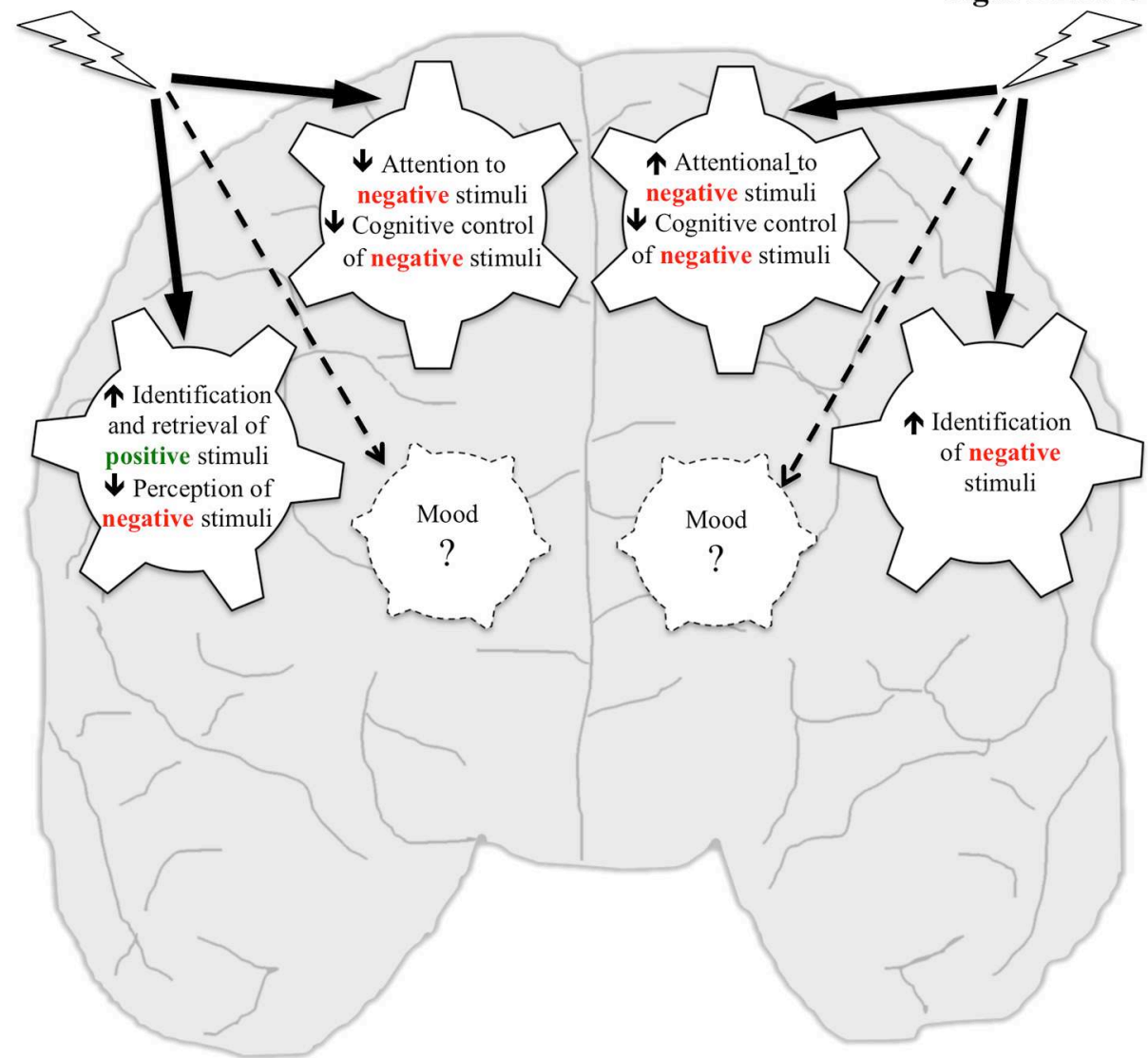

FIGURE 1 | Putative effects of NIBS over the left and right DLPFC on mood, emotional processing, and attentional processing of emotional information in healthy individuals. Wheels represent processes that have been targeted using NIBS. Studies reported that NIBS applied over the left DLPFC increased identification and retrieval of positive stimuli, decreased perception of negative stimuli, decreased attention to negative stimuli, and cognitive control of negative stimuli, but no effect was reported on mood. NIBS applied over the right DLPFC increased identification of negative stimuli, increased attention to negative stimuli and decreased cognitive control of negative stimuli, but no effect was reported on mood.

active rTMS with flipping the coil at a 45 or $90^{\circ}$ angle away from the head, Loo et al., 2000). Third, the DLPFC has been located with several methods. For the rTMS studies, it has been located as the site that is $5 \mathrm{~cm}$ anteriorly from $\mathrm{M} 1$, anatomically defined with the international 10-20 EEG system (F3, F4) or with MRI and stimulated with a neuronavigation system. For the tDCS studies, the DLPFC has been located with the international 1020 EEG system. Therefore, these methodological considerations may have contributed to the seemingly inconsistent results of the studies reviewed here, especially those studying mood in healthy individuals.

Only five studies out of 23 reported that NIBS over the DLPFC modulated mood in healthy subjects. This lack of clear evidence that NIBS can influence mood in healthy individuals differs from studies in individuals with treatment-resistant MDD. Applying high-frequency rTMS over the left DLPFC (O'Reardon et al., 2007), low-frequency over the right DLPFC (Fitzgerald et al., 2003), anodal and cathodal over the left and right
DLPFC respectively (Brunoni et al., 2013) can reduce depressive symptoms in MDD. These differences on the effects of NIBS applied over the DLPFC on mood between healthy individuals and those with MDD may be explained by several factors. First, these populations differed in brain activity, especially within the DLPFC (Martinot et al., 1990), thus NIBS may modulate brain activity differently according to the studied populations. Moreover, studies in MDD delivered several NIBS sessions, whereas most studies in healthy subjects performed single NIBS sessions, which may be insufficient to induce significant mood changes. A meta-analysis analyzing the effects of tDCS in MDD reported a trend for greater mood improvement when more than 10 sessions were delivered as compared to a lesser number (Shiozawa et al., 2014). Here, two studies delivered repeated NIBS sessions in healthy subjects, one reported mood improvement after nine rTMS sessions (Schaller et al., 2011) and one found no change after four tDCS sessions (Motohashi et al., 2013). 
The influence between mood and attention and between mood and cognitive control were not reported in the reviewed articles here focusing on healthy individuals. Interestingly, these findings differ from studies in individuals with MDD, which have suggested a close relationship between mood, emotional processing, and attention. It has even been hypothesized that one mechanism underlying elevated mood in individuals with MDD is that NIBS improve cognitive control and reduce attentional bias for negative stimuli (De Raedt et al., 2014).

To further characterize potential non-specific and selective effects of NIBS over the DLPFC on mood, emotional processing, and attention of emotional information, future studies should identify NIBS-induced neural changes linked to the observed behavioral changes. It is very likely that these effects are not limited to the DLPFC. For instance, De Raedt et al. (2010) showed that engagement and disengagement for angry faces implicated different neural networks. Engagement for angry faces induced activations in the left orbitofrontal cortex, right DLPFC, dorsal/pregenual anterior cingulate cortex (ACC), right superior parietal gyrus (SPG), whereas disengagement for angry faces elicited activations in the right DLPFC, dorsal ACC, and left SPG. Reducing depressive symptoms with rTMS in treatmentresistant MDD also appears to involve a complex neural network. Fox et al. (2012) proposed that targeted DLPFC sites leading to better clinical efficacy were negatively correlated with the subgenual cingulate. It would thus be interesting to investigate NIBS-induced neural changes that are overlapping or not when modulating mood, emotional processing and/or attention of emotional information in healthy individuals.

Another avenue for future investigation is brain state dependency, which is known to play an important role on the effects of NIBS (Lang et al., 2004; Silvanto et al., 2008). Only one study among those reviewed here likely primed the brain

\section{References}

Baeken, C., De Raedt, R., Van Schuerbeek, P., Vanderhasselt, M. A., De Mey, J., Bossuyt, A., et al. (2010). Right prefrontal HF-rTMS attenuates right amygdala processing of negatively valenced emotional stimuli in healthy females. Behav. Brain Res. 214, 450-455. doi: 10.1016/j.bbr.2010.06.029

Baeken, C., Leyman, L., De Raedt, R., Vanderhasselt, M. A., and D’Haenen, H. (2006). Lack of impact of repetitive high frequency transcranial magnetic stimulation on mood in healthy female subjects. J. Affect. Disord. 90, 63-66. doi: 10.1016/j.jad.2005.09.013

Baeken, C., Leyman, L., De Raedt, R., Vanderhasselt, M. A., and D’Haenen, H. (2008). Left and right high frequency repetitive transcranial magnetic stimulation of the dorsolateral prefrontal cortex does not affect mood in female volunteers. Clin. Neurophysiol. 119, 568-575. doi: 10.1016/j.clinph.2007.11.044

Balconi, M., and Cobelli, C. (2015). rTMS on left prefrontal cortex contributes to memories for positive emotional cues: a comparison between pictures and words. Neuroscience 287, 93-103. doi: 10.1016/j.neuroscience.2014.12.015

Balconi, M., and Ferrari, C. (2012a). rTMS stimulation on left DLPFC affects emotional cue retrieval as a function of anxiety level and gender. Depress. Anxiety 29, 976-982. doi: 10.1002/da.21968

Balconi, M., and Ferrari, C. (2012b). rTMS stimulation on left DLPFC increases the correct recognition of memories for emotional target and distractor words. Cogn. Affect. Behav. Neurosci. 12, 589-598. doi: 10.3758/s13415-012-0090-1

Balconi, M., and Ferrari, C. (2013). Repeated transcranial magnetic stimulation on dorsolateral prefrontal cortex improves performance in emotional memory in a specific way. Hoy et al. (2010) presented their subjects with positive affective stimuli when delivering rTMS over the DLPFC. Results were however inconclusive as combination of exposition with rTMS did not modulate mood. Future work might consider guiding brain state when delivering stimulation. Personality traits also seem to influence the effects of NIBS over the DLPFC. NIBS seems to have greater effects in individuals with higher level of anxiety as compared to those with lower level of anxiety on retrieval of positive stimuli (Balconi and Ferrari, 2012b) and in individuals with higher levels of introversion on rating valence of negative stimuli (Peña-Gómez et al., 2011).

In sum, we cannot conclude whether NIBS over the DLPFC can selectively modulate one of these processes based on specific stimulation parameters and whether NIBS modulating a single process influences the others as only one or two of these processes have been studied within a same design. More studies measuring the effects of NIBS on these processes altogether are needed to test whether one can influence the others, or not. The underlying neurocognitive concepts of mood, emotional and attentional processes of emotional information still remain vague. Recent developments of NIBS, along with neuroimaging technics, should contribute to decipher these concepts. Our review highlights the potential for NIBS applied over the DLPFC to modulate emotional processing and attentional processing of emotional information in healthy individuals, whereas its effect on mood remains unclear.

\section{Acknowledgments}

MM holds a Canadian Institutes of Health Research Fellowship Award (CIHR\#140867). SF is supported by the Canada Research Chair in Cognitive Neuroplasticity. retrieval as a function of level of anxiety and stimulus valence. Psychiatry Clin. Neurosci. 67, 210-218. doi: 10.1111/pcn.12041

Bikson, M., and Rahman, A. (2013). Origins of specificity during tDCS: anatomical, activity-selective, and input-bias mechanisms. Front. Hum. Neurosci. 7:688. doi: 10.3389/fnhum.2013.00688

Bouhuys, A. L., Bloem, G. M., and Groothuis, T. G. (1995). Induction of depressed and elated mood by music influences the perception of facial emotional expressions in healthy subjects. J. Affect. Disord. 33, 215-226. doi: 10.1016/0165-0327(94)00092-N

Browning, M., Reid, C., Cowen, P. J., Goodwin, G. M., and Harmer, C. J. (2007). A single dose of citalopram increases fear recognition in healthy subjects. J. Psychopharmacol. 21, 684-960. doi: 10.1177/0269881106074062

Brunoni, A. R., Valiengo, L., Baccaro, A., Zanão, T. A., de Oliveira, J. F., Goulart, A., et al. (2013). The sertraline vs. electrical current therapy for treating depression clinical study: results from a factorial, randomized, controlled trial. JAMA Psychiatry 70, 383-391. doi: 10.1001/2013.jamapsychiatry.32

Brunoni, A. R., Zanao, T. A., Vanderhasselt, M. A., Valiengo, L., de Oliveira, J. F., Boggio, P. S., et al. (2014). Enhancement of affective processing induced by bifrontal transcranial direct current stimulation in patients with major depression. Neuromodulation 17, 138-142. doi: 10.1111/ner.12080

Canli, T., Desmond, J. E., Zhao, Z., Glover, G., and Gabrieli, J. D. (1998). Hemispheric asymmetry for emotional stimuli detected with fMRI. Neuroreport 9, 3233-3239. doi: 10.1097/00001756-199810050-00019

Chepenik, L. G., Cornew, L. A., and Farah, M. J. (2007). The influence of sad mood on cognition. Emotion 7, 802-811. doi: 10.1037/1528-3542.7.4.802 
Clarke, P. J., Browning, M., Hammond, G., Notebaert, L., and Macleod, C. (2014). The causal role of the dorsolateral prefrontal cortex in the modification of attentional bias: evidence from transcranial direct current stimulation. Biol. Psychiatry 76, 946-952. doi: 10.1016/j.biopsych.2014.03.003

Conson, M., Errico, D., Mazzarella, E., Giordano, M., Grossi, D., and Trojano, L. (2015). Transcranial electrical stimulation over dorsolateral prefrontal cortex modulates processing of social cognitive and affective information. PLOS ONE 10:e0126448. doi: 10.1371/journal.pone.0126448

d'Alfonso, A. A., Van Honk, J., Hermans, E., Postma, A., and de Haan, E. H. (2000). Laterality effects in selective attention to threat after repetitive transcranial magnetic stimulation at the prefrontal cortex in female subjects. Neurosci. Lett. 280, 195-198. doi: 10.1016/S0304-3940(00)00781-3

Davidson, R. J., and Irwin, W. (1999). The functional neuroanatomy of emotion and affective style. Trends Cogn. Sci. 3, 11-21. doi: 10.1016/S13646613(98)01265-0

De Raedt, R., Leyman, L., Baeken, C., Van Schuerbeek, P., Luypaert, R., Vanderhasselt, M. A., et al. (2010). Neurocognitive effects of HF-rTMS over the dorsolateral prefrontal cortex on the attentional processing of emotional information in healthy women: an event-related fMRI study. Biol. Psychol. 85, 487-495. doi: 10.1016/j.biopsycho.2010.09.015

De Raedt, R., Vanderhasselt, M. A., and Baeken, C. (2014). Neurostimulation as an intervention for treatment resistant depression: from research on mechanisms towards targeted neurocognitive strategies. Clin. Psychol. Rev. doi: 10.1016/j.cpr.2014.10.006. [Epub ahead of print].

Feeser, M., Prehn, K., Kazzer, P., Mungee, A., and Bajbouj, M. (2014). Transcranial direct current stimulation enhances cognitive control during emotion regulation. Brain Stimul. 7, 105-112. doi: 10.1016/j.brs.2013.08.006

Fitzgerald, P. B., Brown, T. L., Marston, N. A., Daskalakis, Z. J., De Castella, A., and Kulkarni, J. (2003). Transcranial magnetic stimulation in the treatment of depression: a double-blind, placebo-controlled trial. Arch. Gen. Psychiatry 60, 1002-1008. doi: 10.1001/archpsyc.60.9.1002

Fox, M. D., Buckner, R. L., White, M. P., Greicius, M. D., and Pascual-Leone, A. (2012). Efficacy of transcranial magnetic stimulation targets for depression is related to intrinsic functional connectivity with the subgenual cingulate. Biol. Psychiatry 72, 595-603. doi: 10.1016/j.biopsych.2012.04.028

George, M. S., Wassermann, E. M., Williams, W. A., Steppel, J., Pascual-Leone, A., Basser, P., et al. (1996). Changes in mood and hormone levels after rapid-rate transcranial magnetic stimulation (rTMS) of the prefrontal cortex. J. Neuropsychiatry Clin. Neurosci. 8, 172-180. doi: 10.1176/jnp.8.2.172

Grisaru, N., Bruno, R., and Pridmore, S. (2001). Effect on the emotions of healthy individuals of slow repetitive transcranial magnetic stimulation applied to the prefrontal cortex. J. ECT 17, 184-189. doi: 10.1097/00124509-20010900000007

Harmer, C. J., Shelley, N. C., Cowen, P. J., and Goodwin, G. M. (2004). Increased positive versus negative affective perception and memory in healthy volunteers following selective serotonin and norepinephrine reuptake inhibition. Am. J. Psychiatry 161, 1256-1263. doi: 10.1176/appi.ajp.161.7.1256

Herrington, J. D., Mohanty, A., Koven, N. S., Fisher, J. E., Stewart, J. L., Banich, M. T., et al. (2005). Emotion-modulated performance and activity in left dorsolateral prefrontal cortex. Emotion 5, 200-207. doi: 10.1037/15283542.5.2.200

Hoy, K. E., Enticott, P. G., Daskalakis, Z. J., and Fitzgerald, P. B. (2010). Can a behavioral intervention enhance the effect of repetitive transcranial magnetic stimulation on mood? Brain Stimul. 3, 200-206. doi: 10.1016/j.brs.2010.06.001

Jacob, H., Brück, C., Domin, M., Lotze, M., and Wildgruber, D. (2014). I can't keep your face and voice out of my head: neural correlates of an attentional bias toward nonverbal emotional cues. Cereb. Cortex 24, 1460-1473. doi: $10.1093 /$ cercor/bhs417

Lang, N., Siebner, H. R., Ernst, D., Nitsche, M. A., Paulus, W., Lemon, R. N., et al. (2004). Preconditioning with transcranial direct current stimulation sensitizes the motor cortex to rapid-rate transcranial magnetic stimulation and controls the direction of after-effects. Biol. Psychiatry 56, 634-639. doi: 10.1016/j.biopsych.2004.07.017

Leyman, L., De Raedt, R., Vanderhasselt, M. A., and Baeken, C. (2009). Influence of high-frequency repetitive transcranial magnetic stimulation over the dorsolateral prefrontal cortex on the inhibition of emotional information in healthy volunteers. Psychol. Med. 39, 1019-1028. doi: $10.1017 /$ S0033291708004431
Loo, C. K., Taylor, J. L., Gandevia, S. C., McDarmont, B. N., Mitchell, P. B., and Sachdev, P. S. (2000). Transcranial magnetic stimulation (TMS) in controlled treatment studies: are some "sham" forms active? Biol. Psychiatry 47, 325-331. doi: 10.1016/s0006-3223(99)00285-1

MacLeod, C., Rutherford, E., Campbell, L., Ebsworthy, G., and Holker, L. (2002). Selective attention and emotional vulnerability: assessing the causal basis of their association through the experimental manipulation of attentional bias. J. Abnorm. Psychol. 111, 107-123. doi: 10.1037/0021-843X.111.1.107

Martinot, J. L., Hardy, P., Feline, A., Hudet, J. D., Mazoyer, B., Attar-Levy, D., et al. (1990). Left prefrontal glucose hypometabolism in the depressed state: a confirmation. Am. J. Psychiatry 147, 1313-1317. doi: 10.1176/ajp.147.10.1313

Morgan, H. M., Davis, N. J., and Bracewell, R. M. (2014). Does transcranial direct current stimulation to prefrontal cortex affect mood and emotional memory retrieval in healthy individuals? PLOS ONE 9:e92162. doi: 10.1371/journal.pone.0092162

Mosimann, U. P., Rihs, T. A., Engeler, J., Fisch, H., and Schlaepfer, T. E. (2000). Mood effects of repetitive transcranial magnetic stimulation of left prefrontal cortex in healthy volunteers. Psychiatry Res. 94, 251-256. doi: 10.1016/S01651781(00)00146-3

Motohashi, N., Yamaguchi, M., Fujii, T., and Kitahara, Y. (2013). Mood and cognitive function following repeated transcranial direct current stimulation in healthy volunteers: a preliminary report. Neurosci. Res. 77, 64-69. doi: 10.1016/j.neures.2013.06.001

Murphy, S. E., Yiend, J., Lester, K. J., Cowen, P. J., and Harmer, C. J. (2009). Short-term serotonergic but not noradrenergic antidepressant administration reduces attentional vigilance to threat in healthy volunteers. Int. J. Neuropsychopharmacol. 12, 169-179. doi: 10.1017/S1461145708009164

Nitsche, M. A., Koschack, J., Pohlers, H., Hullemann, S., Paulus, W., and Happe, S. (2012). Effects of frontal transcranial direct current stimulation on emotional state and processing in healthy humans. Front. Psychiatry 3:58. doi: $10.3389 /$ fpsyt.2012.00058

O’Reardon, J. P., Solvason, H. B., Janicak, P. G., Sampson, S., Isenberg, K. E., Nahas, Z., et al. (2007). Efficacy and safety of transcranial magnetic stimulation in the acute treatment of major depression: a multisite randomized controlled trial. Biol. Psychiatry 62, 1208-1216. doi: 10.1016/j.biopsych.2007.01.018

Padberg, F., Juckel, G., Prässl, A., Zwanzger, P., Mavrogiorgou, P., Hegerl, U., et al (2001). Prefrontal cortex modulation of mood and emotionally induced facial expressions: a transcranial magnetic stimulation study. J. Neuropsychiatry Clin. Neurosci. 13, 206-212. doi: 10.1176/jnp.13.2.206

Pascual-Leone, A., Catalá, M. D., and Pascual-Leone Pascual, A. (1996). Lateralized effect of rapid-rate transcranial magnetic stimulation of the prefrontal cortex on mood. Neurology 46, 499-502. doi: 10.1212/WNL.46.2.499

Peña-Gómez, C., Vidal-Piñeiro, D., Clemente, I. C., Pascual-Leone, Á., and Bartrés-Faz, D. (2011). Down-regulation of negative emotional processing by transcranial direct current stimulation: effects of personality characteristics. PLoS ONE 6:e22812. doi: 10.1371/journal.pone.0022812

Plazier, M., Joos, K., Vanneste, S., Ost, J., and De Ridder, D. (2012). Bifrontal and bioccipital transcranial direct current stimulation (tDCS) does not induce mood changes in healthy volunteers: a placebo controlled study. Brain Stimul. 5, 454-461. doi: 10.1016/j.brs.2011.07.005

Plewnia, C., Schroeder, P. A., Kunze, R., Faehling, F., and Wolkenstein, L. (2015). Keep calm and carry on: improved frustration tolerance and processing speed by transcranial direct current stimulation (tDCS). PLOS ONE 10:e122578. doi: 10.1371/journal.pone.0122578

Quarto, T., Blasi, G., Pallesen, K. J., Bertolino, A., and Brattico, E. (2014). Implicit processing of visual emotions is affected by sound-induced affective states and individual affective traits. PLOS ONE 9:e103278. doi: 10.1371/journal.pone.0103278

Schaller, G., Lenz, B., Friedrich, K., Dygon, D., Richter-Schmidinger, T., Jacobi, A., et al. (2011). Repetitive transcranial magnetic stimulation influences mood in healthy male volunteers. J. Psychiatr. Res. 45, 1178-1183. doi: 10.1016/j.jpsychires.2011.02.006

Schutter, D. J., van Honk, J., d'Alfonso, A. A., Postma, A., and de Haan, E. H. (2001). Effects of slow rTMS at the right dorsolateral prefrontal cortex on EEG asymmetry and mood. Neuroreport 12, 445-447. doi: 10.1097/00001756200103050-00005

Shafi, M. M., Westover, M. B., Fox, M. D., and Pascual-Leone, A. (2012). Exploration and modulation of brain network interactions with noninvasive 
brain stimulation in combination with neuroimaging. Eur. J. Neurosci. 35, 805-825. doi: 10.1111/j.1460-9568.2012.08035.x

Shiozawa, P., Fregni, F., Benseñor, I. M., Lotufo, P. A., Berlim, M. T., Daskalakis, J. Z., et al. (2014). Transcranial direct current stimulation for major depression: an updated systematic review and meta-analysis. Int. J. Neuropsychopharmacol. 17, 1443-1452. doi: 10.1017/S14611457 14000418

Silvanto, J., Muggleton, N., and Walsh, V. (2008). State-dependency in brain stimulation studies of perception and cognition. Trends Cogn. Sci. 12, 447-454. doi: 10.1016/j.tics.2008.09.004

Steele, J. D., and Lawrie, S. M. (2004). Segregation of cognitive and emotional function in the prefrontal cortex: a stereotactic meta-analysis. Neuroimage 21, 868-875. doi: 10.1016/j.neuroimage.2003.09.066

Vanderhasselt, M. A., Baeken, C., Hendricks, M., and De Raedt, R. (2011). The effects of high frequency rTMS on negative attentional bias are influenced by baseline state anxiety. Neuropsychologia 49, 1824-1830. doi: 10.1016/j.neuropsychologia.2011.03.006

Vanderhasselt, M. A., De Raedt, R., Baeken, C., Leyman, L., Clerinx, P., and D'haenen, H. (2007). The influence of rTMS over the right dorsolateral prefrontal cortex on top-down attentional processes. Brain Res. 1137, 111-116. doi: 10.1016/j.brainres.2006.12.050

Vanderhasselt, M. A., De Raedt, R., Baeken, C., Leyman, L., and D'haenen, H. (2006). The influence of rTMS over the right dorsolateral prefrontal cortex on intentional set switching. Exp. Brain Res. 172, 561-565. doi: 10.1007/s00221006-0540-5

Vanderhasselt, M. A., De Raedt, R., Baeken, C., Leyman, L., and D'Haenen, H. (2009a). A single session of rTMS over the left dorsolateral prefrontal cortex influences attentional control in depressed patients. World J. Biol. Psychiatry 10, 34-42. doi: 10.1080/15622970701816514
Vanderhasselt, M. A., De Raedt, R., Leyman, L., and Baeken, C. (2009b). Acute effects of repetitive transcranial magnetic stimulation on attentional control are related to antidepressant outcomes. J. Psychiatry Neurosci. 34, 119-126.

van Honk, J., Hermans, E. J., d'alfonso, A. A., Schutter, D. J., van Doornen, L., and de Haan, E. H. (2002a). A left-prefrontal lateralized, sympathetic mechanism directs attention towards social threat in humans: evidence from repetitive transcranial magnetic stimulation. Neurosci. Lett. 319, 99-102. doi: 10.1016/S0304-3940(01)02558-7

Van Honk, J., Schutter, D. J., D'alfonso, A. A., Kessels, R. P., and De Haan, E. H. (2002b). $1 \mathrm{hz}$ rTMS over the right prefrontal cortex reduces vigilant attention to unmasked but not to masked fearful faces. Biol. Psychiatry 52, 312-317. doi: 10.1016/S0006-3223(02)01346-X

Wolkenstein, L., and Plewnia, C. (2013). Amelioration of cognitive control in depression by transcranial direct current stimulation. Biol. Psychiatry 73, 646-651. doi: 10.1016/j.biopsych.2012.10.010

Wolkenstein, L., Zeiller, M., Kanske, P., and Plewnia, C. (2014). Induction of a depression-like negativity bias by cathodal transcranial direct current stimulation. Cortex 59, 103-112. doi: 10.1016/j.cortex.2014.07.011

Conflict of Interest Statement: The authors declare that the research was conducted in the absence of any commercial or financial relationships that could be construed as a potential conflict of interest.

Copyright (C) 2015 Mondino, Thiffault and Fecteau. This is an open-access article distributed under the terms of the Creative Commons Attribution License (CC BY). The use, distribution or reproduction in other forums is permitted, provided the original author(s) or licensor are credited and that the original publication in this journal is cited, in accordance with accepted academic practice. No use, distribution or reproduction is permitted which does not comply with these terms. 